\title{
Supplement to: Early Pliocene deepening of the tropical Atlantic thermocline
}

Carolien M. H. van der Weijst ${ }^{1}$, Josse Winkelhorst ${ }^{1}$, Anna von der Heydt ${ }^{2}$, Gert-Jan Reichart ${ }^{1,3}$, Francesca Sangiorgi ${ }^{1}$, Appy Sluijs ${ }^{1}$

${ }^{1}$ Department of Earth Sciences, Utrecht University, 3584 CB Utrecht, the Netherlands ${ }^{2}$ Department of Physics, Utrecht University, 3584CC Utrecht, the Netherlands

${ }^{3}$ NIOZ Royal Netherlands Institute for Sea Research, 1797 SZ ' $t$ Horntje, the Netherlands

Correspondence to: C.M.H. van der Weijst (c.m.h.vanderweijst@uu.nl)

\section{Content}

Figure S1

Figure S2

Site 959 stable oxygen isotope data

References 


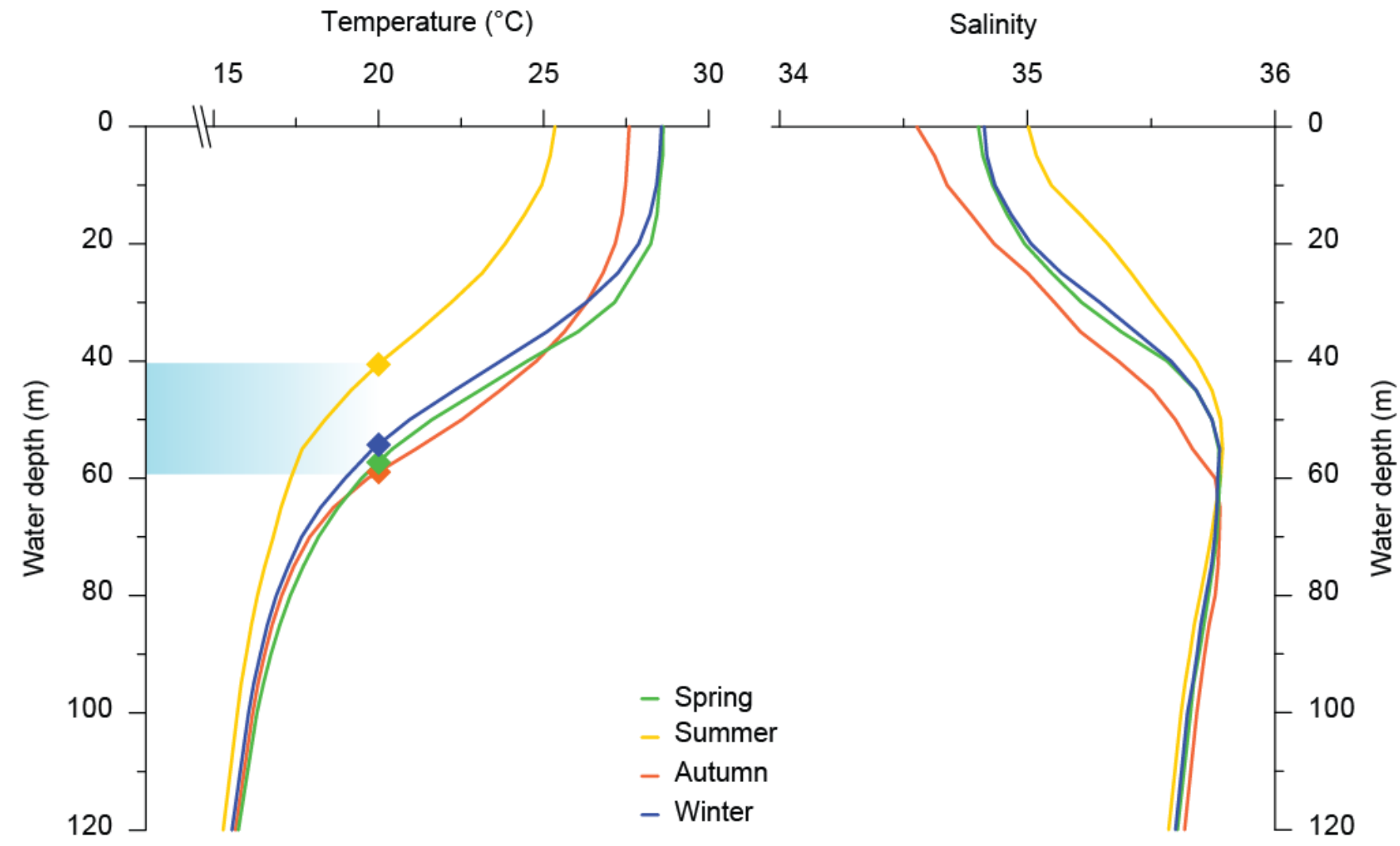

Figure S1: Seasonal vertical temperature and salinity profiles in the Gulf of Guinea $\left(2.5^{\circ} \mathrm{N} 3.5^{\circ} \mathrm{W}\right.$; Locarnini et al., 2013; Zweng et al., 2013). Symbols (left panel) mark the $20^{\circ} \mathrm{C}$ isotherm depth, which varies seasonally between $\sim 40-60$ meters (shaded area). 


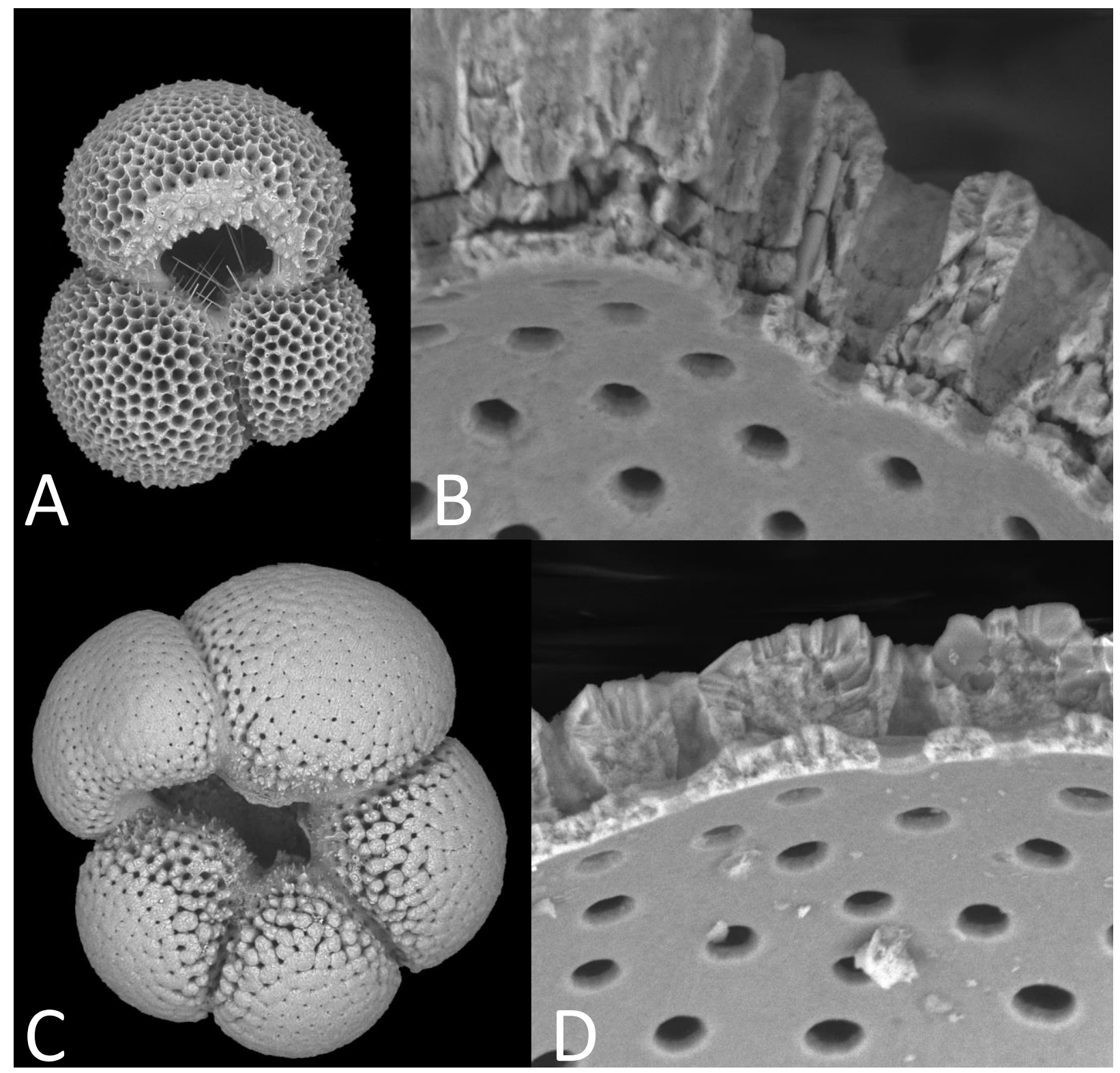

Figure S2. Scanning Electron Microscope pictures of $G$. ruber (panel A and B; sample ODP959C 6H-4, 19-21) and $N$. dutertrei (panel C and D; sample ODP959C 6H-2, 102-104) tests from the late Pliocene interval of ODP Site 959. Whole test photographs taken at $250 \times$ magnification and cross sections at $2500 \times$. 


\begin{tabular}{|c|c|c|c|c|c|c|c|c|c|}
\hline ODP Code & Depth (rMCD) & Age (ka) & $\begin{array}{l}\text { Surface } \delta 180 \\
\text { (\% VPDB) }\end{array}$ & $\begin{array}{l}\text { Surface } \delta 13 \mathrm{C} \\
\text { (\%o VPDB) }\end{array}$ & $\begin{array}{l}\text { Subsurface } \\
\delta 180(\% \circ \\
\text { VPDB) }\end{array}$ & $\begin{array}{l}\text { Subsurface } \\
\delta 13 C(\% \circ \\
\text { VPDB) }\end{array}$ & Surface species & $\begin{array}{l}\text { Subsurface } \\
\text { species }\end{array}$ & Adjustments \\
\hline ODP9595C 5H-2, 77-79 & 35.77 & 2799.3 & -1.38 & 1.70 & & & G. ruber & & \\
\hline ODP9595C 5H-2, 97-99 & 35.97 & 2810.7 & -1.49 & 1.11 & -0.74 & 1.45 & 45 G. ruber & N. dutertrei & \\
\hline ODP9595C 5H-2, 117-119 & 36.17 & 2822.0 & -1.72 & 1.24 & -1.05 & 1.39 & 39 G. ruber & N. dutertrei & \\
\hline ODP9595C 5H-2, 137-139 & 36.37 & 2832.3 & & & -1.06 & 0.53 & 53 & N. dutertrei & \\
\hline ODP9595C 5H-3, 7-9 & 36.58 & 2840.4 & -1.91 & 1.64 & -0.50 & 1.25 & 25 G. ruber & N. dutertrei & \\
\hline ODP9595C 5H-3, 27-29 & 36.78 & 2848.1 & -1.53 & 1.19 & -0.78 & 1.29 & 29 G. ruber & N. dutertrei & \\
\hline ODP9595C 5H-3, 47-49 & 36.98 & 2856.3 & -1.57 & 1.30 & -0.74 & 1.20 & 20 G. ruber & N. dutertrei & \\
\hline ODP9595C 5H-3, 67-69 & 37.18 & 2864.6 & -1.92 & 1.76 & -0.87 & 1.34 & 34 G. ruber & N. dutertrei & \\
\hline ODP9595C 5H-3, 87-89 & 37.38 & 2872.9 & -1.78 & 1.73 & -0.93 & 1.25 & 25 G. ruber & N. dutertrei & \\
\hline ODP9595C 5H-3, 107-109 & 37.58 & 2881.3 & -1.77 & 1.40 & -0.76 & 1.12 & 12 G. ruber & N. dutertrei & \\
\hline ODP9595C 5H-3, 127-129 & 37.77 & 2889.5 & -1.67 & 1.45 & -0.74 & 1.35 & 35 G. ruber & N. dutertrei & \\
\hline ODP9595C 5H-3, 147-149 & 37.96 & 2899.2 & -1.83 & 1.04 & -0.88 & 1.24 & 24 G. ruber & N. dutertrei & \\
\hline ODP9595C 5H-4, 2-4 & 38.00 & 2901.5 & -1.84 & 1.68 & & & G. ruber & & \\
\hline ODP9595C 5H-4, 27-29 & 38.24 & 2915.3 & -1.42 & 0.80 & -0.74 & 1.23 & 23 G. ruber & N. dutertrei & \\
\hline ODP9595C 5H-4, 47-49 & 38.42 & 2925.6 & & & -0.77 & 0.79 & 79 & N. dutertrei & \\
\hline ODP9595C 5H-4, 67-69 & 38.58 & 2934.5 & -1.95 & 1.71 & -0.54 & 1.07 & 07 G. ruber & N. dutertrei & \\
\hline ODP9595C 5H-4, 107-109 & 38.91 & 2951.4 & -1.48 & 1.91 & -0.48 & 1.35 & 35 G. ruber & N. dutertrei & \\
\hline ODP9595C 5H-4, 147-149 & 39.29 & 2971.8 & -1.87 & 1.59 & -0.76 & 1.15 & 15 G. ruber & N. dutertrei & \\
\hline ODP9595C 5H-5, 47-49 & 39.88 & 3006.8 & -1.11 & 1.00 & -0.84 & 0.80 & 80 G. ruber & N. dutertrei & \\
\hline ODP9595C 5H-5, 67-69 & 40.11 & 3018.3 & -1.56 & 1.70 & & & G. ruber & & \\
\hline ODP9595C 5H-5, 87-89 & 40.30 & 3027.6 & -1.59 & 1.00 & -0.90 & 0.98 & 98 G. ruber & N. dutertrei & \\
\hline ODP9595C 5H-5, 107-109 & 40.50 & 3038.3 & -1.94 & 1.24 & -1.02 & 0.97 & 97 G. ruber & N. dutertrei & \\
\hline ODP9595C 5H-5, 127-129 & 40.70 & 3049.3 & & & -0.81 & 0.64 & 64 & N. dutertrei & \\
\hline ODP9595C 5H-5, 147-149 & 40.9 & 3060.3 & & & -1.27 & 0.93 & 93 & N. dutertrei & \\
\hline ODP9595C 5H-6, 27-29 & 41.20 & 3076.9 & -1.94 & 1.64 & -0.95 & 1.03 & 03 G. ruber & N. dutertrei & \\
\hline ODP9595C 5H-6, 32-34 & 41.25 & 3079.6 & -1.86 & 1.49 & -0.98 & 0.86 & 86 G. ruber & N. dutertrei & \\
\hline ODP9595C 5H-6, 47-49 & 41.40 & 3087.9 & -1.66 & 1.79 & -1.16 & 0.76 & 76 G. ruber & N. dutertrei & \\
\hline ODP9595C 5H-6, 52-54 & 41.45 & 3090.7 & -1.67 & 1.69 & -0.91 & 0.91 & 91 G. ruber & N. dutertrei & \\
\hline ODP9595C 5H-6, 67-69 & 41.61 & 3099.5 & -2.30 & 1.02 & -0.82 & 1.19 & 19 G. ruber & N. dutertrei & \\
\hline ODP9595C 5H-6, 87-89 & 41.81 & 3111.5 & -2.21 & 1.60 & -1.19 & 0.34 & 34 G. ruber & N. dutertrei & \\
\hline ODP9595C 5H-6, 107-109 & 42.01 & 3124.6 & -1.73 & 1.42 & & & G. ruber & & \\
\hline ODP9595C 5H-6, 127-129 & 42.21 & 3136.6 & -1.73 & 1.12 & -0.83 & 1.03 & 03 G. ruber & N. dutertrei & \\
\hline ODP9595C 5H-6, 147-149 & 42.41 & 3144.6 & -1.24 & 0.86 & -0.89 & 0.34 & 34 G. ruber & N. dutertrei & \\
\hline ODP9595C 5H-7, 7-9 & 42.52 & 3149.0 & -1.42 & 1.05 & -0.80 & 0.56 & 56 G. ruber & N. dutertrei & \\
\hline ODP9595C 5H-7, 12-14 & 42.57 & 3151.0 & -1.83 & 1.42 & -1.03 & 0.84 & 84 G. ruber & N. dutertrei & \\
\hline ODP9595C 5H-7, 22-24 & 42.67 & 3155.0 & -2.15 & 1.62 & -1.39 & 0.30 & 30 G. ruber & N. dutertrei & \\
\hline ODP9595C 5H-7, 27-29 & 42.72 & 3157.0 & -2.21 & 2.05 & -1.11 & 0.68 & 68 G. ruber & N. dutertrei & \\
\hline ODP9595C 5H-7, 37-39 & 42.83 & 3161.3 & -1.75 & 1.56 & & & G. ruber & & \\
\hline ODP9595C 5H-7, 40-42 & 42.84 & 3161.7 & -1.77 & 1.59 & -0.45 & 1.23 & 23 G. ruber & N. dutertrei & \\
\hline ODP9595C 5H-7, 47-49 & 42.93 & 3165.2 & -1.93 & 1.28 & -0.53 & 1.03 & 03 G. ruber & N. dutertrei & \\
\hline ODP9595C 5H-7, 62-64 & 43.07 & 3170.7 & -1.85 & 1.72 & & & G. ruber & & \\
\hline ODP9595C 6H-1, 7-9 & 43.11 & 3172.3 & -1.69 & 1.54 & -0.66 & 1.01 & 01 G. ruber & N. dutertrei & \\
\hline ODP9595C 6H-1, 12-14 & 43.16 & 3174.3 & -1.67 & 1.62 & -0.70 & 1.11 & 11 G. ruber & N. dutertrei & \\
\hline ODP9595C 6H-1, 22-24 & 43.26 & 3178.2 & -1.68 & 1.56 & -0.90 & 1.09 & 09 G. ruber & N. dutertrei & \\
\hline ODP9595C 6H-1, 37-39 & 43.41 & 3184.1 & -1.61 & 1.50 & -0.60 & 1.30 & 30 G. ruber & N. dutertrei & \\
\hline ODP9595C 6H-1, 42-44 & 43.46 & 3186.1 & -1.91 & 2.14 & -0.44 & 1.33 & 33 G. ruber & N. dutertrei & \\
\hline ODP9595C 6H-1, 47-49 & 43.51 & 3188.0 & -1.84 & 1.66 & -0.50 & 1.32 & 32 G. ruber & N. dutertrei & \\
\hline ODP9595C 6H-1, 57-59 & 43.61 & 3192.1 & -2.19 & 1.65 & -0.64 & 1.22 & 22 G. ruber & N. dutertrei & \\
\hline ODP9595C 6H-1, 60-62 & 43.63 & 3192.9 & -1.60 & 2.03 & -0.49 & 1.35 & 35 G. ruber & N. dutertrei & \\
\hline ODP9595C 6H-1, 62-64 & 43.66 & 3194.1 & -1.62 & 2.09 & -0.49 & 1.21 & 21 G. ruber & N. dutertrei & \\
\hline ODP9595C 6H-1, 67-69 & 43.71 & 3196.2 & -1.46 & 1.99 & -0.58 & 1.14 & 14 G. ruber & N. dutertrei & \\
\hline ODP9595C 6H-1, 82-84 & 43.86 & 3202.4 & -1.71 & 1.86 & -0.71 & 1.24 & 24 G. ruber & N. dutertrei & \\
\hline ODP9595C 6H-1, 102-104 & 44.06 & 3210.6 & -1.65 & 1.88 & -0.35 & 1.12 & 12 G. ruber & N. dutertrei & \\
\hline ODP9595C 6H-1, 107-109 & 44.11 & 3212.6 & -1.66 & 1.74 & -0.44 & 1.25 & 25 G. ruber & N. dutertrei & \\
\hline ODP9595C 6H-1, 117-119 & 44.21 & 3216.8 & -1.29 & 2.13 & -0.56 & 1.09 & 09 G. ruber & N. dutertrei & \\
\hline ODP9595C 6H-1, 122-124 & 44.26 & 3218.8 & -1.90 & 1.86 & -0.55 & 1.27 & 27 G. ruber & N. dutertrei & \\
\hline ODP9595C 6H-1, 127-129 & 44.31 & 3220.9 & -1.58 & 1.50 & & & G. ruber & & \\
\hline ODP9595C 6H-1, 132-134 & 44.36 & 3222.9 & -1.48 & 1.99 & & & G. ruber & & \\
\hline ODP9595C 6H-1, 142-144 & 44.46 & 3227.9 & & & -0.61 & 1.27 & 27 & N. dutertrei & \\
\hline ODP9595C 6H-2, 7-9 & 44.61 & 3236.4 & -1.76 & 1.48 & -0.64 & 1.04 & 04 G. ruber & N. dutertrei & \\
\hline ODP9595C 6H-2, 12-14 & 44.66 & 3239.3 & -1.94 & 1.88 & -0.59 & 1.09 & 09 G. ruber & N. dutertrei & \\
\hline ODP9595C 6H-2, 17-19 & 44.71 & 3242.1 & & & -0.24 & 1.05 & 05 & N. dutertrei & \\
\hline ODP9595C 6H-2, 22-24 & 44.76 & 3245.0 & -1.93 & 1.65 & -0.24 & 1.26 & 26 G. ruber & N. dutertrei & \\
\hline ODP9595C 6H-2, 32-34 & 44.86 & 3258.3 & -1.84 & 1.75 & -0.75 & 0.89 & 89 G. ruber & N. dutertrei & \\
\hline ODP9595C 6H-2, 37-39 & 44.91 & 3265.0 & -1.67 & 1.61 & -0.52 & 1.22 & 22 G. ruber & N. dutertrei & \\
\hline ODP9595C 6H-2, 45-47 & 44.98 & 3267.3 & -1.75 & 1.39 & -0.48 & 1.13 & 13 G. ruber & N. dutertrei & \\
\hline ODP9595C 6H-2, 52-54 & 45.06 & 3270.0 & -1.60 & 1.81 & -0.68 & 1.15 & 15 G. ruber & N. dutertrei & \\
\hline ODP9595C 6H-2, 60-62 & 45.16 & 3273.3 & -1.67 & 1.44 & -0.43 & 1.20 & 20 G. ruber & N. dutertrei & \\
\hline ODP9595C 6H-2, 62-64 & 45.21 & 3275.0 & -1.75 & 1.61 & -0.10 & 1.12 & 12 G. ruber & N. dutertrei & \\
\hline ODP9595C 6H-2, 67-69 & 45.26 & 3276.7 & -1.90 & 1.84 & -0.45 & 1.30 & 30 G. ruber & N. dutertrei & \\
\hline ODP9595C 6H-2, 77-79 & 45.31 & 3278.3 & -1.71 & 1.79 & -0.72 & 1.07 & 07 G. ruber & N. dutertrei & \\
\hline ODP9595C 6H-2, 82-84 & 45.36 & 3280.0 & -1.65 & 1.58 & -0.27 & 1.40 & 40 G. ruber & N. dutertrei & \\
\hline ODP9595C 6H-2, 92-94 & 45.46 & 3287.3 & -1.45 & 1.53 & -0.50 & 0.99 & 99 G. ruber & N. dutertrei & \\
\hline ODP9595C 6H-2, 102-104 & 45.56 & 3294.5 & -1.53 & 1.19 & & & G. ruber & & \\
\hline ODP9595C 6H-2, 107-109 & 45.61 & 3298.2 & -1.43 & 1.20 & & & G. ruber & & \\
\hline ODP9595C 6H-2, 112-114 & 45.66 & 3301.8 & -1.59 & 1.56 & -0.27 & 0.76 & 76 G. ruber & N. dutertrei & \\
\hline ODP9595C 6H-2, 117-119 & 45.71 & 3305.5 & -1.55 & 1.25 & & & G. ruber & & \\
\hline ODP9595C 6H-2, 120-122 & 45.73 & 3306.9 & -1.58 & 1.23 & -0.30 & 0.90 & 90 G. ruber & N. dutertrei & \\
\hline ODP9595C 6H-2, 122-124 & 45.76 & 3309.1 & -2.18 & 1.46 & -0.63 & 0.90 & 90 G. ruber & N. dutertrei & \\
\hline ODP9595C 6H-2, 127-129 & 45.81 & 3312.7 & -1.82 & 1.48 & -0.26 & 0.95 & 95 G. ruber & N. dutertrei & \\
\hline ODP9595C 6H-2, 132-134 & 45.86 & 3316.4 & -1.96 & 1.67 & -0.19 & 1.12 & 12 G. ruber & N. dutertrei & \\
\hline ODP9595C 6H-2, 137-139 & 45.91 & 3320.0 & -1.76 & 1.64 & -0.40 & 1.19 & 19 G. ruber & N. dutertrei & \\
\hline ODP9595C 6H-2, 142-144 & 45.96 & 3321.9 & -1.84 & 1.41 & -0.45 & 1.04 & 04 G. ruber & N. dutertrei & \\
\hline ODP9595C 6H-2, 147-149 & 46.01 & 3323.7 & -1.54 & 1.29 & -0.71 & 0.75 & 75 G. ruber & N. dutertrei & \\
\hline ODP9595C 6H-3, 6-8 & 46.10 & 3327.0 & -1.78 & 1.68 & -0.55 & 0.95 & 95 G. ruber & N. dutertrei & \\
\hline ODP9595C 6H-3, 21-23 & 46.25 & 3332.6 & -1.82 & 1.39 & -0.12 & 1.17 & 17 G. ruber & N. dutertrei & \\
\hline ODP9595C 6H-3, 41-43 & 46.45 & 3340.0 & -1.69 & 0.97 & -0.77 & 1.02 & 02 G. ruber & N. dutertrei & \\
\hline $9595 \mathrm{C} 6 \mathrm{H}-3$ & 4 & 33 & -1.68 & 1. & -0 & 34 & 94 G. $r$ & dute & \\
\hline
\end{tabular}




\begin{tabular}{|c|c|c|c|c|c|c|}
\hline ODP9595C 6H-3, 81-83 & 46.85 & 3371.4 & -1.82 & 0.80 & -0.53 & 0.90 G. ruber \\
\hline ODP9595C 6H-3, 101-103 & 47.05 & 3384.5 & -1.65 & 0.49 & -0.42 & 0.82 G. ruber \\
\hline ODP9595C 6H-3, 121-123 & 47.25 & 3402.5 & -1.80 & 1.37 & -0.54 & 0.90 G. ruber \\
\hline ODP9595C 6H-3, 141-143 & 47.45 & 3420.5 & -1.81 & 1.31 & -0.43 & 0.62 G. ruber \\
\hline ODP9595C 6H-4, 19-21 & 47.73 & 3440.0 & -1.95 & 1.65 & -0.67 & 0.97 G. ruber \\
\hline ODP9595C 6H-4, 24-26 & 47.78 & 3442.9 & -2.09 & 1.64 & -0.83 & 0.84 G. ruber \\
\hline ODP9595C 6H-4, 29-31 & 47.83 & 3445.7 & -1.91 & 1.74 & -0.86 & 1.20 G. ruber \\
\hline ODP9595C 6H-4, 30-32 & 47.84 & 3446.3 & -1.80 & 1.66 & -0.91 & 1.10 G. ruber \\
\hline ODP9595C 6H-4, 59-61 & 48.13 & 3462.9 & -1.87 & 1.34 & -0.48 & 0.90 G. ruber \\
\hline ODP9595C 6H-4, 79-81 & 48.33 & 3474.3 & -1.81 & 0.83 & & G. ruber \\
\hline ODP9595C 6H-4, 99-101 & 48.53 & 3484.9 & -1.99 & 1.41 & -0.66 & 1.20 G. ruber \\
\hline ODP9595C 6H-4, 119-121 & 48.73 & 3494.6 & -1.87 & 1.50 & -1.03 & 1.20 G. ruber \\
\hline See Norris (1998) & 52.43 & 3675.1 & & & -0.70 & 1.16 \\
\hline See Norris (1998) & 55.12 & 3806.4 & & & -0.66 & 1.18 \\
\hline See Norris (1998) & 55.32 & 3816.1 & -1.68 & 2.15 & -0.42 & 1.17 G. sacculifer \\
\hline See Norris (1998) & 55.52 & 3825.9 & -1.72 & 2.32 & -0.67 & 1.25 G. sacculifer \\
\hline See Norris (1998) & 55.65 & 3832.2 & -1.46 & 2.01 & -0.40 & 0.71 G. sacculifer \\
\hline See Norris (1998) & 55.78 & 3838.6 & -1.15 & 1.63 & -0.71 & 0.96 G. sacculifer \\
\hline See Norris (1998) & 55.98 & 3847.0 & -1.43 & 2.01 & -0.70 & 0.93 G. sacculifer \\
\hline See Norris (1998) & 56.18 & 3854.8 & -1.15 & 1.90 & -0.78 & 1.19 G. sacculifer \\
\hline See Norris (1998) & 56.28 & 3858.6 & -1.88 & 2.51 & -0.66 & 0.91 G. sacculifer \\
\hline See Norris (1998) & 56.48 & 3866.4 & -1.38 & 1.90 & -0.66 & 0.97 G. sacculifer \\
\hline See Norris (1998) & 56.68 & 3874.1 & -1.26 & 2.03 & -0.60 & 0.99 G. sacculifer \\
\hline See Norris (1998) & 56.88 & 3881.8 & -1.15 & 2.03 & -0.67 & 1.03 G. sacculifer \\
\hline See Norris (1998) & 57.08 & 3889.6 & -1.97 & 2.19 & -0.67 & 0.89 G. sacculifer \\
\hline See Norris (1998) & 57.28 & 3897.3 & -1.47 & 2.21 & -0.67 & 0.94 G. sacculifer \\
\hline See Norris (1998) & 57.48 & 3905.0 & -1.28 & 1.88 & -0.69 & 1.03 G. sacculifer \\
\hline See Norris (1998) & 57.68 & 3912.8 & -1.64 & 2.22 & -0.53 & 0.98 G. sacculifer \\
\hline See Norris (1998) & 57.78 & 3916.6 & -1.42 & 2.01 & -0.85 & 0.85 G. sacculifer \\
\hline See Norris (1998) & 57.98 & 3924.4 & -1.64 & 1.99 & -0.65 & 0.84 G. sacculifer \\
\hline See Norris (1998) & 58.18 & 3932.1 & -1.52 & 1.87 & -0.90 & 0.60 G. sacculifer \\
\hline See Norris (1998) & 58.38 & 3939.9 & -1.28 & 1.71 & -0.88 & 0.71 G. sacculifer \\
\hline See Norris (1998) & 58.58 & 3947.6 & -1.18 & 1.54 & -0.66 & 0.76 G. sacculifer \\
\hline See Norris (1998) & 58.98 & 3963.1 & -1.80 & 2.19 & -1.17 & 0.60 G. sacculifer \\
\hline See Norris (1998) & 59.18 & 3970.8 & -1.64 & 1.89 & -0.83 & 0.65 G. sacculifer \\
\hline See Norris (1998) & 59.28 & 3974.7 & -1.97 & 2.02 & -0.55 & 1.03 G. sacculifer \\
\hline See Norris (1998) & 59.48 & 3982.4 & -1.33 & 1.85 & -0.89 & 0.54 G. sacculifer \\
\hline See Norris (1998) & 59.68 & 3990.1 & -1.30 & 1.77 & -0.95 & 0.72 G. sacculifer \\
\hline See Norris (1998) & 59.88 & 3997.9 & -1.11 & 1.57 & -0.62 & 0.57 G. sacculifer \\
\hline See Norris (1998) & 60.08 & 4005.6 & -2.25 & 2.00 & -0.75 & 0.97 G. sacculifer \\
\hline See Norris (1998) & 60.28 & 4013.3 & -1.72 & 2.18 & -0.60 & 1.02 G. sacculifer \\
\hline See Norris (1998) & 60.48 & 4021.1 & -1.52 & 1.64 & -1.17 & 0.80 G. sacculifer \\
\hline See Norris (1998) & 60.68 & 4028.8 & -1.43 & 1.66 & -0.49 & 0.61 G. sacculifer \\
\hline See Norris (1998) & 60.78 & 4032.7 & -2.54 & 1.93 & -0.67 & 0.70 G. sacculifer \\
\hline See Norris (1998) & 60.98 & 4040.4 & -1.96 & 1.89 & -0.78 & 0.90 G. sacculifer \\
\hline See Norris (1998) & 61.18 & 4048.2 & -1.16 & 1.58 & -0.71 & 0.88 G. sacculifer \\
\hline See Norris (1998) & 61.38 & 4055.9 & -1.41 & 2.47 & -0.66 & 0.97 G. sacculifer \\
\hline See Norris (1998) & 61.58 & 4063.6 & -1.58 & 2.32 & -0.76 & 1.06 G. sacculifer \\
\hline See Norris (1998) & 61.78 & 4071.4 & -1.71 & 2.09 & -0.48 & 0.79 G. sacculifer \\
\hline See Norris (1998) & 61.98 & 4079.5 & -1.87 & 2.38 & -0.65 & 0.86 G. sacculifer \\
\hline See Norris (1998) & 62.18 & 4088.2 & -1.71 & 2.29 & -0.58 & 1.14 G. sacculifer \\
\hline See Norris (1998) & 62.28 & 4092.6 & -1.31 & 2.37 & -0.84 & 0.86 G. sacculifer \\
\hline See Norris (1998) & 62.47 & 4100.9 & -1.24 & 2.00 & -0.39 & 1.00 G. sacculifer \\
\hline See Norris (1998) & 62.63 & 4107.8 & -1.24 & 2.14 & -0.78 & 0.63 G. sacculifer \\
\hline See Norris (1998) & 62.79 & 4114.8 & -0.61 & 1.54 & -0.62 & 0.78 G. sacculifer \\
\hline See Norris (1998) & 62.93 & 4120.9 & -1.07 & 1.93 & -0.72 & 0.82 G. sacculifer \\
\hline See Norris (1998) & 63.05 & 4126.2 & -1.89 & 2.07 & -0.90 & 1.09 G. sacculifer \\
\hline See Norris (1998) & 63.19 & 4132.3 & -1.83 & 2.13 & -0.81 & 1.09 G. sacculifer \\
\hline See Norris (1998) & 63.32 & 4137.9 & -2.11 & 2.00 & -0.69 & 1.13 G. sacculifer \\
\hline See Norris (1998) & 63.38 & 4140.7 & -2.03 & 2.46 & -0.91 & 1.06 G. sacculifer \\
\hline See Norris (1998) & 63.51 & 4146.2 & -1.68 & 2.16 & -1.22 & 0.93 G. sacculifer \\
\hline See Norris (1998) & 63.64 & 4151.9 & -1.28 & 1.35 & -0.92 & 0.72 G. sacculifer \\
\hline See Norris (1998) & 63.71 & 4154.9 & -1.60 & 2.02 & -1.07 & 0.96 G. sacculifer \\
\hline See Norris (1998) & 63.78 & 4158.0 & -1.22 & 2.06 & -0.80 & 0.54 G. sacculifer \\
\hline See Norris (1998) & 68.27 & 4353.8 & -1.69 & 2.25 & -0.72 & 1.04 G. sacculifer \\
\hline See Norris (1998) & 68.33 & 4356.6 & -1.50 & 2.28 & -0.59 & 0.99 G. sacculifer \\
\hline See Norris (1998) & 68.48 & 4363.0 & -1.91 & 2.15 & -0.93 & 0.68 G. sacculifer \\
\hline See Norris (1998) & 68.61 & 4368.7 & -1.98 & 2.22 & -0.64 & 0.78 G. sacculifer \\
\hline See Norris (1998) & 68.81 & 4377.4 & -2.14 & 2.08 & -1.07 & 0.79 G. sacculifer \\
\hline See Norris (1998) & 69.00 & 4385.7 & -1.56 & 1.72 & -0.77 & 0.86 G. sacculifer \\
\hline See Norris (1998) & 69.05 & 4387.6 & -1.45 & 1.96 & -0.79 & 0.94 G. sacculifer \\
\hline See Norris (1998) & 69.14 & 4391.8 & -1.88 & 2.14 & -0.77 & 0.67 G. sacculifer \\
\hline See Norris (1998) & 69.26 & 4397.0 & -1.85 & 2.20 & -0.66 & 1.07 G. sacculifer \\
\hline See Norris (1998) & 69.41 & 4403.6 & -1.55 & 2.27 & -1.14 & 0.93 G. sacculifer \\
\hline See Norris (1998) & 69.63 & 4413.2 & -1.88 & 2.21 & -0.94 & 0.85 G. sacculifer \\
\hline See Norris (1998) & 69.85 & 4422.8 & -1.59 & 2.23 & -0.72 & 0.91 G. sacculifer \\
\hline See Norris (1998) & 70.07 & 4432.4 & -1.82 & 1.97 & -0.94 & 1.04 G. sacculifer \\
\hline See Norris (1998) & 70.33 & 4443.7 & -1.42 & 1.91 & -0.84 & 0.96 G. sacculifer \\
\hline See Norris (1998) & 70.46 & 4449.2 & -1.70 & 1.95 & -0.59 & 0.70 G. sacculifer \\
\hline See Norris (1998) & 70.68 & 4459.0 & -1.75 & 2.17 & -0.37 & 0.76 G. sacculifer \\
\hline See Norris (1998) & 70.84 & 4465.9 & -1.76 & 2.37 & -0.58 & 0.65 G. sacculifer \\
\hline See Norris (1998) & 71.00 & 4472.9 & -2.10 & 2.40 & -0.61 & 0.70 G. sacculifer \\
\hline See Norris (1998) & 71.16 & 4479.9 & -1.72 & 2.18 & -0.66 & 0.60 G. sacculifer \\
\hline See Norris (1998) & 71.51 & 4495.2 & -1.45 & 2.22 & -0.72 & 0.62 G. sacculifer \\
\hline See Norris (1998) & 71.77 & 4506.5 & -1.54 & 2.03 & -0.77 & 0.61 G. sacculifer \\
\hline See Norris (1998) & 72.03 & 4517.8 & -2.28 & 2.24 & -0.94 & 0.82 G. sacculifer \\
\hline See Norris (1998) & 72.20 & 4525.3 & -1.90 & 1.71 & -1.35 & 0.71 G. sacculifer \\
\hline See Norris (1998) & 72.28 & 4528.8 & & & -0.64 & 0.60 \\
\hline See Norris (1998) & 72.48 & 4537.5 & -1.12 & 2.27 & -0.17 & 0.57 G. sacculifer \\
\hline See Norris (1998) & 72.56 & 4541.0 & -1.51 & 2.17 & -0.71 & 0.89 G. sacculifer \\
\hline See Norris (1998) & 72.64 & 4544.5 & -1.45 & 2.07 & -0.91 & culifer \\
\hline $\mathrm{S}$ & 72.76 & 4549.7 & -1.77 & 2.17 & 1.10 & 0.73 G. sacculifer \\
\hline
\end{tabular}




\begin{tabular}{|c|c|c|c|c|c|c|}
\hline See Norris (1998) & 72.89 & 4555.4 & -0.52 & 1.34 & -0.28 & 0.31 G. sacculifer \\
\hline See Norris (1998) & 72.96 & 4558.6 & -1.57 & 2.09 & -0.46 & $0.41 \mathrm{G}$. sacculifer \\
\hline See Norris (1998) & 73.14 & 4566.3 & -1.68 & 2.55 & 1.02 & 0.75 G. sacculifer \\
\hline See Norris (1998) & 73.34 & 4575.0 & -2.17 & 2.26 & 0.99 & 0.92 G. sacculifer \\
\hline See Norris (1998) & 73.57 & 4585.0 & -1.98 & 2.09 & -0.76 & 1.16 G. sacculifer \\
\hline See Norris (1998) & 73.78 & 4594.2 & -1.95 & 1.84 & 0.48 & 0.62 G. sacculifer \\
\hline See Norris (1998) & 73.93 & 4600.7 & -1.50 & 2.02 & -0.79 & 0.82 G. sacculifer \\
\hline See Norris (1998) & 74.08 & 4607.3 & -1.16 & 1.21 & 0.27 & 0.43 G. sacculifer \\
\hline See Norris (1998) & 74.23 & 4613.8 & -1.83 & 1.83 & 0.63 & 0.66 G. sacculifer \\
\hline See Norris (1998) & 74.35 & 4619.0 & -2.24 & 1.82 & -0.17 & 0.59 G. sacculifer \\
\hline See Norris (1998) & 74.58 & 4629.1 & -2.16 & 1.91 & 0.84 & 0.81 G. sacculifer \\
\hline See Norris (1998) & 74.79 & 4638.2 & -1.47 & 1.95 & 0.55 & 0.75 G. sacculifer \\
\hline See Norris (1998) & 74.98 & 4646.5 & -1.97 & 1.84 & -0.10 & 0.64 G. sacculifer \\
\hline See Norris (1998) & 75.17 & 4654.8 & -1.70 & 2.16 & 0.71 & 0.78 G. sacculifer \\
\hline See Norris (1998) & 75.36 & 4663.1 & -1.86 & 2.28 & 0.42 & 0.68 G. sacculifer \\
\hline See Norris (1998) & 75.48 & 4668.3 & -1.96 & 2.24 & -0.39 & 0.86 G. sacculifer \\
\hline See Norris (1998) & 75.63 & 4674.9 & -1.95 & 2.23 & 0.32 & $0.61 \mathrm{G}$. sacculifer \\
\hline See Norris (1998) & 76.12 & 4696.2 & -1.78 & 2.20 & 0.17 & 0.81 G. sacculifer \\
\hline See Norris (1998) & 76.34 & 4705.8 & -1.50 & 1.97 & -0.57 & 0.85 G. sacculifer \\
\hline See Norris (1998) & 76.45 & 4710.6 & -2.11 & 2.19 & -0.12 & 0.76 G. sacculifer \\
\hline See Norris (1998) & 76.50 & 4712.8 & -1.88 & 2.18 & 0.54 & 0.88 G. sacculifer \\
\hline See Norris (1998) & 76.56 & 4715.4 & -2.02 & 2.18 & -0.32 & 1.06 G. sacculifer \\
\hline See Norris (1998) & 76.61 & 4717.3 & -1.84 & 2.30 & 0.19 & 0.75 G. sacculifer \\
\hline See Norris (1998) & 79.55 & 4831.6 & -1.53 & 2.13 & 0.28 & 0.77 G. sacculifer \\
\hline See Norris (1998) & 79.75 & 4839.3 & -1.69 & 2.07 & 0.63 & 0.57 G. sacculifer \\
\hline See Norris (1998) & 79.95 & 4847.1 & -1.80 & 2.20 & 0.50 & 0.88 G. sacculifer \\
\hline See Norris (1998) & 80.15 & 4857.5 & -1.86 & 1.99 & 0.37 & 0.80 G. sacculifer \\
\hline See Norris (1998) & 80.35 & 4870.6 & -2.15 & 2.46 & 0.37 & 0.87 G. sacculifer \\
\hline See Norris (1998) & 80.55 & 4883.7 & -1.84 & 2.31 & -0.43 & 0.74 G. sacculifer \\
\hline See Norris (1998) & 80.65 & 4890.3 & -2.05 & 1.95 & 0.92 & $0.73 \mathrm{G}$. sacculifer \\
\hline See Norris (1998) & 80.85 & 4903.4 & -1.43 & 1.55 & 0.83 & 0.51 G. sacculifer \\
\hline See Norris (1998) & 81.05 & 4916.5 & -1.82 & 2.39 & 0.51 & 0.68 G. sacculifer \\
\hline See Norris (1998) & 81.25 & 4929.6 & -1.74 & 2.58 & -0.69 & 0.88 G. sacculifer \\
\hline See Norris (1998) & 81.45 & 4942.7 & -1.85 & 2.38 & -0.39 & 0.29 G. sacculifer \\
\hline See Norris (1998) & 81.65 & 4955.7 & -2.18 & 2.28 & -0.62 & 0.80 G. sacculifer \\
\hline See Norris (1998) & 81.85 & 4968.8 & -1.42 & 2.21 & -0.48 & 0.54 G. sacculifer \\
\hline See Norris (1998) & 82.05 & 4981.9 & -1.96 & 2.14 & -0.60 & 0.55 G. sacculifer \\
\hline See Norris (1998) & 82.15 & 4988.5 & -2.18 & 2.63 & -0.86 & 0.71 G. sacculifer \\
\hline See Norris (1998) & 82.35 & 5001.6 & -2.00 & 2.41 & -0.17 & 0.62 G. sacculifer \\
\hline See Norris (1998) & 82.55 & 5014.7 & -1.50 & 2.38 & -0.81 & 0.68 G. sacculifer \\
\hline See Norris (1998) & 82.75 & 5027.8 & -2.09 & 2.29 & -1.00 & 1.05 G. sacculifer \\
\hline See Norris (1998) & 82.95 & 5040.8 & -1.79 & 2.29 & -1.08 & 0.33 G. sacculifer \\
\hline See Norris (1998) & 83.15 & 5053.9 & -1.97 & 2.13 & -1.19 & 0.67 G. sacculifer \\
\hline See Norris (1998) & 83.35 & 5067.0 & -2.21 & 2.01 & -0.77 & 0.68 G. sacculifer \\
\hline See Norris (1998) & 83.55 & 5075.8 & -2.30 & 2.65 & -0.48 & 0.61 G. sacculifer \\
\hline See Norris (1998) & 83.65 & 5079.8 & -2.01 & 2.36 & 0.56 & 0.74 G. sacculifer \\
\hline See Norris (1998) & 83.85 & 5087.7 & -2.33 & 2.74 & 0.20 & 0.63 G. sacculifer \\
\hline See Norris (1998) & 84.05 & 5095.7 & -2.14 & 1.94 & -0.52 & 0.39 G. sacculifer \\
\hline See Norris (1998) & 84.25 & 5103.7 & -1.21 & 1.82 & -1.00 & 0.27 G. sacculifer \\
\hline See Norris (1998) & 84.45 & 5111.6 & -1.25 & 1.69 & -0.73 & 0.37 G. sacculifer \\
\hline See Norris (1998) & 84.65 & 5119.6 & -2.17 & 2.10 & -1.05 & 0.99 G. sacculifer \\
\hline See Norris (1998) & 84.85 & 5127.6 & -1.80 & 2.39 & -0.31 & 0.77 G. sacculifer \\
\hline See Norris (1998) & 85.05 & 5135.5 & -1.60 & 1.74 & -0.72 & $0.41 \mathrm{G}$. sacculifer \\
\hline See Norris (1998) & 85.15 & 5139.5 & -1.63 & 2.10 & -0.69 & 0.76 G. sacculifer \\
\hline See Norris (1998) & 85.35 & 5147.5 & -1.69 & 2.21 & -0.52 & 0.24 G. sacculifer \\
\hline See Norris (1998) & 85.55 & 5155.4 & -1.64 & 1.90 & -0.72 & 0.37 G. sacculifer \\
\hline See Norris (1998) & 85.75 & 5163.4 & -2.03 & 2.29 & -0.10 & 0.78 G. sacculifer \\
\hline See Norris (1998) & 85.95 & 5171.4 & -1.77 & 2.26 & -1.00 & 0.89 G. sacculifer \\
\hline See Norris (1998) & 86.15 & 5179.3 & -1.97 & 2.29 & 0.15 & 0.80 G. sacculifer \\
\hline See Norris (1998) & 86.35 & 5187.3 & -2.01 & 2.23 & 0.35 & 0.67 G. sacculifer \\
\hline See Norris (1998) & 86.55 & 5195.3 & -1.74 & 1.99 & 0.27 & 0.46 G. sacculifer \\
\hline See Norris (1998) & 86.65 & 5199.3 & -1.48 & 2.30 & 0.63 & 0.63 G. sacculifer \\
\hline See Norris (1998) & 86.85 & 5207.2 & -1.66 & 2.06 & 0.48 & 0.39 G. sacculifer \\
\hline See Norris (1998) & 87.05 & 5215.2 & -1.73 & 2.05 & 0.81 & 0.94 G. sacculifer \\
\hline See Norris (1998) & 87.25 & 5227.2 & -2.35 & 2.40 & 0.66 & 1.02 G. sacculifer \\
\hline See Norris (1998) & 87.45 & 5239.6 & -2.07 & 2.32 & 0.24 & 0.59 G. sacculifer \\
\hline See Norris (1998) & 87.65 & 5251.9 & -2.10 & 2.35 & 0.50 & 0.71 G. sacculifer \\
\hline See Norris (1998) & 87.85 & 5264.3 & -1.95 & 2.28 & -0.14 & 0.51 G. sacculifer \\
\hline See Norris (1998) & 88.05 & 5276.7 & -1.63 & 2.59 & 0.43 & 0.70 G. sacculifer \\
\hline See Norris (1998) & 88.14 & 5282.5 & -1.68 & 2.20 & -0.36 & 0.52 G. sacculifer \\
\hline See Norris (1998) & 88.32 & 5293.5 & -1.61 & 2.23 & 0.16 & 0.73 G. sacculifer \\
\hline See Norris (1998) & 88.49 & 5304.0 & -1.64 & 2.34 & -0.16 & 0.43 G. sacculifer \\
\hline See Norris (1998) & 88.58 & 5309.6 & -1.86 & 2.42 & -0.06 & 0.63 G. sacculifer \\
\hline See Norris (1998) & 88.67 & 5315.2 & -1.92 & 2.30 & 0.38 & 0.69 G. sacculifer \\
\hline See Norris (1998) & 88.75 & 5320.1 & -1.77 & 2.03 & 0.18 & 0.44 G. sacculifer \\
\hline See Norris (1998) & 88.95 & 5332.5 & -1.95 & 2.06 & 1.01 & $1.03 \mathrm{G}$. sacculifer \\
\hline See Norris (1998) & 89.16 & 5345.5 & -1.36 & 2.28 & 0.55 & 1.03 G. sacculifer \\
\hline See Norris (1998) & 89.36 & 5357.9 & -2.30 & 2.20 & 0.37 & 0.67 G. sacculifer \\
\hline See Norris (1998) & 89.57 & 5370.9 & -2.01 & 2.12 & 0.59 & 0.67 G. sacculifer \\
\hline See Norris (1998) & 89.78 & 5382.2 & -1.60 & 2.04 & 0.57 & 0.76 G. sacculifer \\
\hline See Norris (1998) & 89.98 & 5390.0 & -1.89 & 2.52 & 0.55 & 1.05 G. sacculifer \\
\hline See Norris (1998) & 90.08 & 5394.0 & -1.99 & 1.88 & 0.02 & 0.80 G. sacculifer \\
\hline See Norris (1998) & 90.29 & 5402.3 & -1.93 & 2.19 & 0.78 & 0.83 G. sacculifer \\
\hline See Norris (1998) & 90.50 & 5410.5 & -1.60 & 2.22 & 0.13 & 0.64 G. sacculifer \\
\hline See Norris (1998) & 90.70 & 5418.4 & -1.75 & 2.13 & 0.53 & 0.81 G. sacculifer \\
\hline See Norris (1998) & 90.91 & 5426.7 & -2.13 & 2.18 & 0.09 & 0.80 G. sacculifer \\
\hline See Norris (1998) & 91.11 & 5434.6 & -1.85 & 2.83 & 1.01 & 1.16 G. sacculifer \\
\hline See Norris (1998) & 91.32 & 5442.8 & -1.90 & 2.63 & 0.81 & 0.89 G. sacculifer \\
\hline See Norris (1998) & 91.50 & 5449.9 & -1.69 & 2.52 & 0.64 & $1.01 \mathrm{G}$. sacculifer \\
\hline See Norris (1998) & 91.59 & 5453.3 & -2.16 & 2.36 & 0.70 & 0.80 G. sacculifer \\
\hline (1998) & 91.78 & 5460.7 & -1.61 & 1.99 & 0.50 & 0.69 G. sacculifer \\
\hline See Norris (1998) & 91.96 & 5467.6 & -1.84 & 2.3 & 0.59 & 0.76 G. sacculifer \\
\hline
\end{tabular}




\begin{tabular}{|c|c|c|c|c|c|c|c|c|c|}
\hline See Norris (1998) & 92.15 & 5475.0 & -2.06 & 2.03 & 0.81 & 0.49 G. sacculifer & N. dutertrei & G. sacculifer $-0.33 \%$ & Norris (1998) \\
\hline See Norris (1998) & 92.35 & 5482.7 & -1.98 & 2.21 & 0.67 & 0.75 G. sacculifer & N. dutertrei & G. sacculifer $-0.33 \%$ & Norris (1998) \\
\hline See Norris (1998) & 92.55 & 5490.4 & -1.58 & 1.66 & 0.75 & 0.47 G. sacculifer & N. dutertrei & G. sacculifer $-0.33 \%$ & Norris (1998) \\
\hline See Norris (1998) & 92.75 & 5498.2 & -2.01 & 2.31 & 0.70 & 0.64 G. sacculifer & N. dutertrei & G. sacculifer $-0.33 \%$ & Norris (1998) \\
\hline See Norris (1998) & 92.95 & 5505.9 & -1.88 & 2.22 & 0.15 & 0.61 G. sacculifer & N. dutertrei & G. sacculifer $-0.33 \%$ & Norris (1998) \\
\hline See Norris (1998) & 93.05 & 5509.8 & -1.70 & 2.52 & 0.70 & 0.85 G. sacculifer & N. dutertrei & G. sacculifer $-0.33 \%$ & Norris (1998) \\
\hline See Norris (1998) & 93.25 & 5517.5 & -1.60 & 2.36 & 1.15 & 0.92 G. sacculifer & N. dutertrei & G. sacculifer $-0.33 \%$ & Norris (1998) \\
\hline See Norris (1998) & 93.45 & 5525.2 & -1.90 & 2.62 & 0.98 & 0.81 G. sacculifer & N. dutertrei & G. sacculifer $-0.33 \%$ & Norris (1998) \\
\hline See Norris (1998) & 93.65 & 5533.0 & -1.47 & 2.45 & 0.96 & 1.20 G. sacculifer & N. dutertrei & G. sacculifer $-0.33 \%$ & Norris (1998) \\
\hline See Norris (1998) & 93.85 & 5540.7 & -2.09 & 2.32 & 0.51 & 0.70 G. sacculifer & N. dutertrei & G. sacculifer $-0.33 \%$ & Norris (1998) \\
\hline See Norris (1998) & 94.05 & 5548.4 & -2.06 & 2.45 & 0.64 & 0.92 G. sacculifer & N. dutertrei & G. sacculifer $-0.33 \%$ & Norris (1998) \\
\hline See Norris (1998) & 94.25 & 5556.2 & -1.82 & 2.07 & 1.06 & 0.83 G. sacculifer & N. dutertrei & G. sacculifer $-0.33 \%$ & Norris (1998) \\
\hline See Norris (1998) & 94.45 & 5563.9 & -2.35 & 2.40 & 0.67 & 0.74 G. sacculifer & N. dutertrei & G. sacculifer $-0.33 \%$ & Norris (1998) \\
\hline See Norris (1998) & 94.55 & 5567.7 & -2.34 & 2.05 & 0.57 & 0.60 G. sacculifer & N. dutertrei & G. sacculifer $-0.33 \%$ & Norris (1998) \\
\hline See Norris (1998) & 94.75 & 5575.5 & -1.42 & 2.32 & 0.93 & 0.95 G. sacculifer & N. dutertrei & G. sacculifer $-0.33 \%$ & Norris (1998) \\
\hline See Norris (1998) & 94.95 & 5583.2 & -1.67 & 2.54 & 1.39 & 0.80 G. sacculifer & N. dutertrei & G. sacculifer $-0.33 \%$ & Norris (1998) \\
\hline See Norris (1998) & 95.15 & 5590.9 & -1.61 & 2.37 & 1.13 & 0.78 G. sacculifer & N. dutertrei & G. sacculifer $-0.33 \%$ & Norris (1998) \\
\hline See Norris (1998) & 95.35 & 5598.7 & -1.85 & 2.25 & 0.75 & 0.78 G. sacculifer & N. dutertrei & G. sacculifer $-0.33 \%$ & Norris (1998) \\
\hline See Norris (1998) & 95.55 & 5606.4 & -1.32 & 2.29 & 0.92 & 0.79 G. sacculifer & N. dutertrei & G. sacculifer $-0.33 \%$ & Norris (1998) \\
\hline See Norris (1998) & 95.75 & 5614.1 & -1.63 & 2.14 & 1.02 & 0.66 G. sacculifer & N. dutertrei & G. sacculifer $-0.33 \%$ & Norris (1998) \\
\hline See Norris (1998) & 95.85 & 5618.0 & -1.55 & 2.47 & 0.97 & 0.78 G. sacculifer & N. dutertrei & G. sacculifer $-0.33 \%$ & Norris (1998) \\
\hline See Norris (1998) & 95.95 & 5621.9 & -1.83 & 2.37 & 0.79 & 0.86 G. sacculifer & N. dutertrei & G. sacculifer $-0.33 \%$ & Norris (1998) \\
\hline See Norris (1998) & 96.05 & 5625.7 & -1.74 & 2.52 & 1.04 & 0.82 G. sacculifer & N. dutertrei & G. sacculifer $-0.33 \%$ & Norris (1998) \\
\hline See Norris (1998) & 96.25 & 5633.4 & -1.59 & 2.24 & 1.07 & 1.02 G. sacculifer & N. dutertrei & G. sacculifer $-0.33 \%$ & Norris (1998) \\
\hline See Norris (1998) & 96.45 & 5641.2 & -2.00 & 1.90 & 0.93 & 0.86 G. sacculifer & N. dutertrei & G. sacculifer $-0.33 \%$ & Norris (1998) \\
\hline See Norris (1998) & 96.69 & 5650.5 & -1.63 & 2.72 & 0.83 & 0.76 G. sacculifer & N. dutertrei & G. sacculifer $-0.33 \%$ & Norris (1998) \\
\hline See Norris (1998) & 96.95 & 5659.8 & -1.64 & 2.64 & 1.01 & 1.05 G. sacculifer & N. dutertrei & G. sacculifer $-0.33 \%$ & Norris (1998) \\
\hline See Norris (1998) & 97.17 & 5667.6 & -1.65 & 2.44 & 0.86 & 1.00 G. sacculifer & N. dutertrei & G. sacculifer $-0.33 \%$ & Norris (1998) \\
\hline
\end{tabular}




\begin{tabular}{lrrr} 
rmcd & $\delta 180$ (G.sacculifer) & $\delta 180$ (G.ruber) & \multicolumn{1}{l}{ Offset } \\
38.00 & -1.49 & -1.84 & -0.35 \\
38.24 & -1.31 & -1.42 & -0.12 \\
39.88 & -1.42 & -1.11 & 0.32 \\
41.81 & -1.46 & -2.21 & -0.75 \\
43.11 & -1.18 & -1.69 & -0.50 \\
43.71 & -1.68 & -1.46 & 0.21 \\
44.11 & -1.34 & -1.66 & -0.32 \\
44.31 & -1.56 & -1.58 & -0.02 \\
44.66 & -1.37 & -1.94 & -0.57 \\
44.86 & -1.37 & -1.84 & -0.47 \\
45.16 & -1.61 & -1.67 & -0.06 \\
45.36 & -1.24 & -1.65 & -0.41 \\
45.56 & -1.21 & -1.53 & -0.32 \\
45.86 & -1.09 & -1.96 & -0.87 \\
46.45 & -1.34 & -1.69 & -0.34 \\
46.85 & -1.32 & -1.82 & -0.50 \\
47.05 & -1.05 & -1.65 & -0.60 \\
47.73 & -1.66 & -1.95 & -0.29 \\
47.83 & -1.75 & -1.91 & -0.16 \\
48.13 & -1.43 & -1.87 & -0.44 \\
48.33 & -0.93 & -1.81 & -0.88
\end{tabular}

average $\quad-0.35$

stdev 0.31




\section{References}

Locarnini, R. A., Mishonov, A. V., Antonov, J. I., Boyer, T. P., Garcia, H. E., Baranova, O. K., Zweng, M. M., Paver, C. R., Reagan, J. R., Johnson, D. R., Hamilton, M., and Seidov, D.: World Ocean Atlas 2013, Volume 1: Temperature, edited by: Levitus, S., NOAA Atlas NESDIS, 73, 40 pp., 2013.

Norris, R.D.: Miocene-Pliocene surface-water hydrography of the eastern equatorial Atlantic. In: Mascle, J., Lohmann, G.P., Moullade, M. (eds.), Proceedings of the Ocean Drilling Program, Scientific Results Vol. 159, College Station, TX (Ocean Drilling Program), 539-555, 1998.

Vallé, F., Westerhold, T. and Dupont, L.: Orbital-driven environmental changes recorded at ODP Site 959 (eastern equatorial Atlantic) from the Late Miocene to the Early Pleistocene, Int. J. Earth Sci., 106(3), 11611174, doi:10.1007/s00531-016-1350-z, 2016.

Zweng, M. M., Reagan, J. R., Antonov, J. I., Locarnini, R. A., Mishonov, A. V., Boyer, T. P., Garcia, H. E., Baranova, O. K., Johnson, D. R., Seidov, D., and Biddle, M. M.: World Ocean Atlas 2013, Volume 2: Salinity, edited by: Levitus, S., NOAA Atlas NESDIS, 74, 39 pp., 2013. 